\title{
A phase II trial of Xeloda and oxaliplatin (XELOX) neo-adjuvant chemotherapy followed by surgery for advanced gastric cancer patients with para-aortic lymph node metastasis
}

\author{
Yan Wang $\cdot$ Yi-yi Yu $\cdot$ Wei Li $\cdot$ Yi Feng $\cdot$ Jun Hou \\ Yuan Ji $\cdot$ Yi-hong Sun $\cdot$ Kun-tang Shen · \\ Zhen-bin Shen · Xin-yu Qin · Tian-shu Liu
}

Received: 8 January 2014 / Accepted: 11 March 2014 / Published online: 21 April 2014

(C) The Author(s) 2014. This article is published with open access at Springerlink.com

\begin{abstract}
Purpose Gastric cancer with para-aortic lymph node (PAN) involvement is regarded as advanced disease, and only chemotherapy is recommended from the guidelines. In unresectable cases, neoadjuvant chemotherapy could prolong survival if conversion to resectability could be achieved.

Methods The study was a single-arm phase II trial. Patients who were diagnosed with gastric cancer and PAN involvement (Stations No. 16a2/16b1) were treated with capecitabine and oxaliplatin combination chemotherapy every 3 weeks for a maximum of six cycles. After every two cycles, abdominal computed tomographic scans were repeated to evaluate the response, and surgery was performed at the physician's discretion in patients with sufficient tumor response, followed by chemotherapy with the same regimen to complete a total of six cycles. The primary end point was the response rate of the preoperative chemotherapy. The secondary end points were R0 resection rate, progression-free survival (PFS), overall survival (OS), and adverse events.
\end{abstract}

Yan Wang and Yiyi Yu have contributed equally to this study.

Y. Wang $\cdot$ Y. Yu $\cdot$ W. Li $\cdot$ Y. Feng $\cdot$ T. Liu $(\bowtie)$

Department of Medical Oncology, Fudan University Zhongshan Hospital, 180 Fenglin Road, Shanghai 200032, People's Republic of China

e-mail: flyingwyan@gmail.com; liu.tianshu@zs-hospital.sh.cn

J. Hou · Y. Ji

Department of Pathology, Fudan University Zhongshan Hospital,

Shanghai 200032, People's Republic of China

Y. Sun $\cdot$ K. Shen $\cdot$ Z. Shen $\cdot$ X. Qin

Department of General Surgery, Fudan University Zhongshan

Hospital, Shanghai 200032, People's Republic of China
Results A total of 48 patients were enrolled. The response rate of the first-line chemotherapy was $49.0 \%$, and the clinical benefit response was $85.1 \%$. After a median of four cycles of chemotherapy, 28 patients received surgery $(58.3 \%)$. The median PFS and OS of all patients were 10.0 and 29.8 months, respectively. Patients in the surgery group had much longer PFS (18.1 vs. $5.6 \mathrm{mo}, P=0.001$ ) and $\mathrm{OS}$ (not reached vs. $12.5 \mathrm{mo}$, $P=0.016)$ compared with those in the non-surgery group.

Conclusions For gastric cancer patients with PAN involvement, neoadjuvant chemotherapy with XELOX demonstrated a good response rate, and a sufficient R0 resection rate, with acceptable toxicities. Further study is needed to confirm the effectiveness of this regimen.

Keywords Gastric cancer - Para-aortic lymph node . Chemotherapy

\section{Introduction}

Despite a decrease in incidence in recent decades, gastric cancer is still one of the most common causes of cancer deaths of worldwide. In general, gastric cancer patients are diagnosed late, with high frequency of nodal involvement [1]. In advanced gastric cancer patients who underwent radical surgery, the incidence of microscopic metastasis in the para-aortic lymph node (PAN) region has been reported to range from 10 to $30 \%$ [2]. The PAN region is defined as the terminal nodes of the stomach, and termed N3 nodes by Japanese Classification of Gastric Carcinoma [3], and as M1 nodes according to the International Union Against Cancer (UICC) tumor-node-metastasis (TNM) classification [4]. 
Gastric cancer with PAN metastasis is often unresectable and has a poor prognosis even after an R0 resection and extensive lymph node dissection. Furthermore, it has been reported previously that there is no survival benefit from surgery and D2 lymphadenectomy with para-aortic node dissection (D2 + PAND). The 5-year overall survival (OS) rate was found to be similar in the groups assigned to D2 lymphadenectomy alone and D2 +PAND $(P=0.85)$ [5]. The median OS of patients with PAN involvement was 13.8 months after palliative chemotherapy without surgery [6].

Some investigators have reported that potentially curable gastric cancer can be successfully treated with neoadjuvant chemotherapy [7]. Several chemotherapy regimens have been introduced in an attempt to downstage the tumor, and prolong survival following by curative resection $[8,9]$. However, few previous reports have documented chemotherapy that enables the curative resection of gastric cancer with PAN involvement.

The objective of this study is to determine whether palliative chemotherapy in patients with gastric cancer and PAN involvement could make subsequent radical surgery feasible and improve OS.

\section{Patients and methods}

\section{Patient selection}

Patients were enrolled who had gastric or gastroesophageal adenocarcinoma proven histologically and PAN metastasis. The histological diagnosis was established by upper gastrointestinal endoscopic biopsies in all cases. The T-stage was determined by endoscopic ultrasonography (EUS), and $\mathrm{N}$-stage was determined by abdominal computed tomographic (CT) scanning. Chest and pelvis CT scanning were performed to rule out distant metastases. PAN was defined as nodes in the region between the upper margin of the celiac artery and the upper border of the inferior mesenteric artery (Stations No. 16a2/16b1), and with diameters $>1.0 \mathrm{~cm}$ by abdominal CT scanning. In addition, eligible patients were required to have an Eastern Cooperative Oncology Group performance status of $0-1$.

Patients were excluded if they had peritoneal gross metastasis, lung metastasis, liver metastasis, pleural effusion, other distant metastasis, or serious uncontrolled comorbid conditions. Patients who could not comprehend or comply with the study were also ineligible. A multidisciplinary evaluation was required before a patient's participation in this study. All patients signed an approved written informed consent form. The protocol of this trial was approved by the institutional review board of Zhongshan Hospital, Fudan University.
Chemotherapy schedule

Chemotherapy was given as the first-line treatment. The regimen of the chemotherapy was as follows: XELOX: capecitabine of $1,000 \mathrm{mg} / \mathrm{m}^{2}$ (orally administered twice a day on days 1-14) and oxaliplatin at $130 \mathrm{mg} / \mathrm{m}^{2}$ (on day 1 , as intravenous $2 \mathrm{~h}$ boluses). The regimens were repeated every 3 weeks after initiation of the first cycle. Six cycles were administered during perioperative period, or treatment was stopped when disease progression was observed.

\section{Tumor response and toxicity criteria}

After every two cycles, an abdominal CT scan was performed to evaluate the response. When PAN metastasis disappeared or shrank to $<1.0 \mathrm{~cm}$, surgery was considered. If surgery could not be done, chemotherapy would be continued until evidence of disease progression appeared. If operation could not be done after six cycles of chemotherapy, the patients would not have the opportunity for operation, and the treatment was stopped. The response to the treatment was evaluated according to response evaluation criteria for solid tumors (RECIST) 1.1 [10]. Pathological complete response (path CR) was defined as an absence of carcinoma cells in the primary site, and pathological partial response (path PR) was defined as $<10 \%$ residual carcinoma cells in the specimen [11]. Adverse events were assessed according to the Common Toxicity Criteria of the National Cancer Institute (NCI-CTC) 3.0 [12].

\section{Surgical procedure}

A staging laparoscopy was performed to reveal whether there were peritoneal metastases in the patients with sufficient response to be considered for subsequent radical surgery. If there was no peritoneal metastasis, radical surgery could be done. The type of surgery performed depended on the location and extent of the primary cancer. The cancer was resected along with a gastric margin of $\geq 5 \mathrm{~cm}$ when feasible. For distal cancers, a subtotal gastrectomy was considered, and total gastrectomy or total esophagogastrectomy was performed for proximal cancers. An attempt was made to perform a D2-type nodal dissection.

\section{Postoperative treatment}

After R0 resection, adjuvant chemotherapy with the XELOX regimen was initiated within 42 days of surgery, and six cycles were administered during perioperative period. Patients who could not undergo a radical operation, continued original chemotherapy until evidence of disease 
progression appeared. If patients were confirmed to have progressive disease, palliative second-line chemotherapy was administered to those patients who could tolerate it. Adjuvant radiotherapy was not administered after R0 resection. All enrolled patients were followed up regularly. Physical and blood examinations were conducted every 3 months for the first 3 years and every 6 months thereafter. An abdominal CT was performed every 6 months for the first 3 years and every year thereafter. Chest CT and upper gastrointestinal endoscopy were conducted every year.

Study design and statistical analysis

This trial investigated the efficacy and safety of preoperative XELOX followed by surgery in gastric cancer patients with PAN involvement. The primary study end point was the response rate. Secondary end points were R0 resection rate, progression-free survival (PFS), OS, and adverse events.

PFS was measured from the date of initial treatment to the first objective documentation of disease progression or relapse. OS was measured from the start of the treatment to the date of the last follow-up or death. In this trial, the sample size was 48 cases, which provided $80 \%$ power based on the hypothesis as the expected value of $80 \%$, and a threshold value of $65 \%$ in the primary end point using one-sided testing at a $10 \%$ significance level. All patients were followed up every 3 months. The PFS and OS were generated by the Kaplan-Meier method.

\section{Results}

Baseline characteristics

From November 2008 to January 2013, 48 patients were enrolled in the study. The median age was 63.5 years (range from 35 to 77 years). Other baseline characteristics of the patients are shown in Table 1.

Response to the chemotherapy (Table 2)

Patients received a median of four cycles of chemotherapy regimens. Forty-seven patients had responses ultimately (one did not because of acute perforation of stomach 5 days after the first regimen of chemotherapy). Two persons had a complete response (CR), 21 had partial responses (PR), 17 had stable disease (SD), and seven had progression of disease (PD). The response rate (CR + PR) was $49 \%$ (23/47), and clinical benefit response $(\mathrm{CP}+\mathrm{PR}+\mathrm{SD})$ was $85.1 \%$ (40/47). The response evaluations of patients with or without surgery are shown in Table 2.
Table 1 Baseline characteristics $(N=48)$

\begin{tabular}{lll}
\hline Clinical features & $N$ & $\%$ \\
\hline Gender $(N, \%)$ & 41 & 85.4 \\
Male & 7 & 14.6 \\
Female & $63.5(35-77)$ & \\
Age (median year, range) & & 16.7 \\
Location $(N, \%)$ & 8 & 83.3 \\
AEG & 40 & 50 \\
G & & 37.5 \\
Lauren type $(N, \%)$ & 24 & 12.5 \\
Intestinal type & 18 & \\
Diffuse type & 6 & 45.8 \\
Mixed type & & 54.2 \\
CEA $(N, \%)$ & 22 & \\
Normal & 26 & 77.1 \\
Elevated & & \\
Anemia & 37 & \\
Present $(N, \%)$ &
\end{tabular}

$A E G$ adenocarcinoma of esophagogastric junction, $G$ gastric cancer

Table 2 Response evaluation after chemotherapy

\begin{tabular}{llr}
\hline & Number of patients $(N)$ & $\%$ \\
\hline Response evaluation & $n=47^{\mathrm{a}}$ & \\
CR & 2 & 4.26 \\
PR & 21 & 44.68 \\
SD & 17 & 36.17 \\
PD & 7 & 14.89 \\
Response of patients received & $n=28$ & \\
surgery & & \\
CR & 0 & 0 \\
PR & 20 & 71.43 \\
SD & 5 & 17.86 \\
PD & 2 & 7.14 \\
a & 1 & 3.57 \\
Response of patients who did not & $n=20$ & \\
receive surgery & & 10.00 \\
CR & 2 & 5.00 \\
PR & 1 & 60.00 \\
SD & 12 & 25.00 \\
PD & 5 &
\end{tabular}

a One did not have response evaluation because of acute perforation of stomach 5 days after the first regimen of chemotherapy and had palliative surgery quickly

Surgical findings and pathology staging (Table 3)

After response evaluation, patients whose PAN metastasis disappeared or shrank to $<1.0 \mathrm{~cm}$ were considered for surgery. A staging laparoscopy was performed first, and three patients were diagnosed with peritoneal carcinomatosis. 
Table 3 Surgical findings after chemotherapy

\begin{tabular}{llr}
\hline & Number of patients $(N)$ & $\%$ \\
\hline Patients received surgery & $n=28$ & \\
Surgery type & & \\
Radical surgery & 24 & 85.71 \\
Palliative surgery & 4 & 14.29 \\
Pathological response & $n=28$ & \\
Responders & 17 & 60.71 \\
Non-responders & 11 & 39.30 \\
pCR & 3 & 10.70 \\
T-stage after surgery & $n=28$ & \\
yT0 & 3 & 10.71 \\
yT1 & 2 & 7.15 \\
yT2 & 5 & 17.86 \\
yT3 & 6 & 21.43 \\
yT4a & 9 & 32.14 \\
yT4b & 3 & 10.71 \\
Patients with positive lymph nodes & $n=28$ & \\
0 & 8 & 28.57 \\
$1-2$ & 3 & 10.71 \\
$3-6$ & 4 & 14.29 \\
$\geq 7$ & 13 & 46.43 \\
\hline
\end{tabular}

Then, palliative surgery was provided. At last twenty-eight patients received surgery $(58.3 \%$, four had palliative gastrectomy, and 24 had radical gastrectomy and D2 lymphadenectomy), and 20 patients (41.7\%) did not receive operation. In the four patients who received palliative surgery, one had an acute perforation of stomach, and the other three had peritoneal metastases. Second-line treatment was administered after R2 operation. In the 20 patients who did not receive surgery after six cycles of chemotherapy, two had $\mathrm{CR}$, and soon afterward stopped treatment. One had PR, but refused operation, and the original treatment was continued. Twelve had SD, and continued on first-line chemotherapy until there was evidence of disease progression. Five patients who developed progressive disease did not have second-line treatment because of poor performance status.

In the operation group, 17 cases $(60.71 \%)$ had pathological responses, and three of them had complete pathological responses $(10.7 \%)$. The median time from surgery to discharge was 9 days (range from 7 to 15 days). Only one of the 28 patients had postoperative complications described as lung infection after surgery.

Survival

After a median follow-up of 12.4 months (range 3.3$58.7 \mathrm{mo}$ ), 20 patients died, 23 patients had disease progression, and nine patients relapsed. In the nine patients who

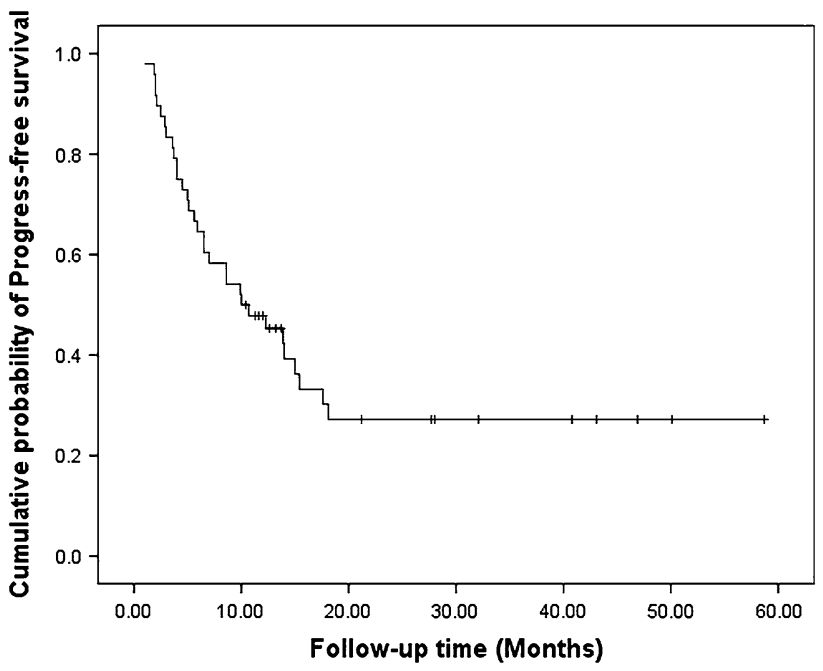

Fig. 1 Progress free survival of all patients $(n=48)$

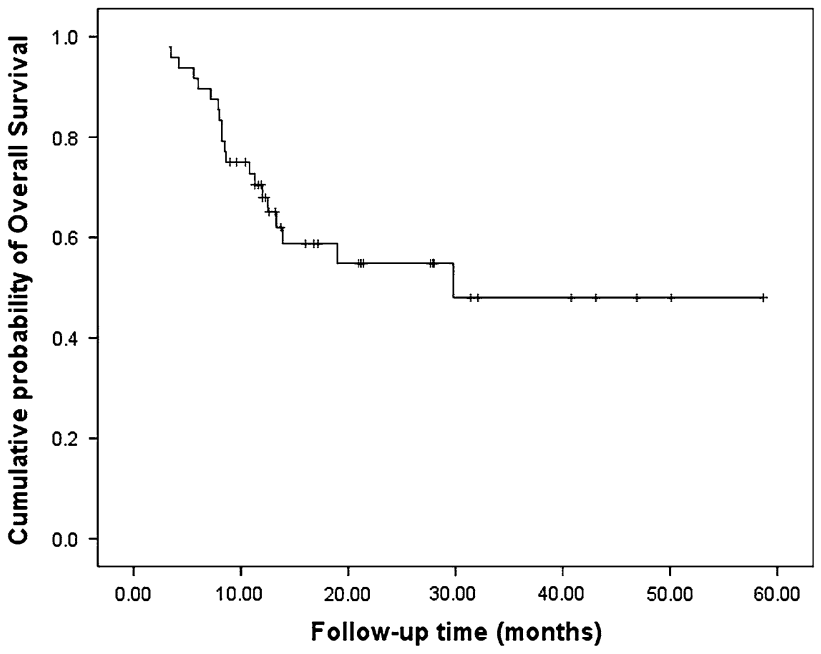

Fig. 2 Overall survival of all patients $(n=48)$

relapsed, one had lung metastasis, one had liver metastasis, two had bone metastases, two had local recurrences, and the other three had left supraclavicular lymph node metastases. The median PFS and OS were 10.0 months (Fig. 1) and 29.8 months, respectively (Fig. 2). The 1-year PFS rate was $47.8 \%$ and 1-year survival rate was $67.9 \%$. Patients in the surgery group had much longer PFS (18.1 vs. 5.6 mo, $P=0.001$ ) and OS (not reached vs. $12.5 \mathrm{mo}, P=0.016$ ) compared with the non-surgery group (Figs. 3, 4).

Toxicity

As shown in Table 4, the most common adverse events were gastrointestinal issues and leukocytopenia. However, only one patient had grade 3 gastrointestinal toxicity and 


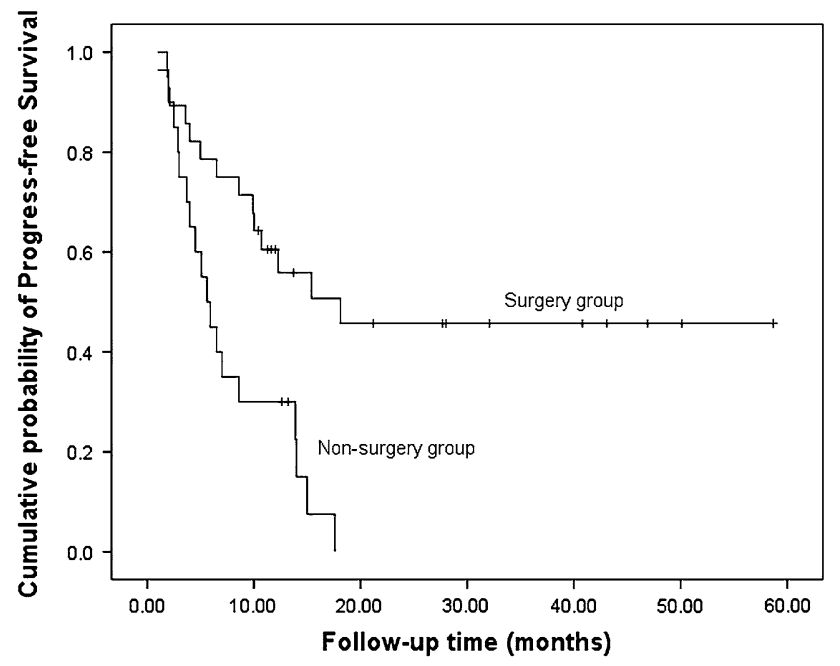

Fig. 3 Progress free survival of surgery group and non-surgery group

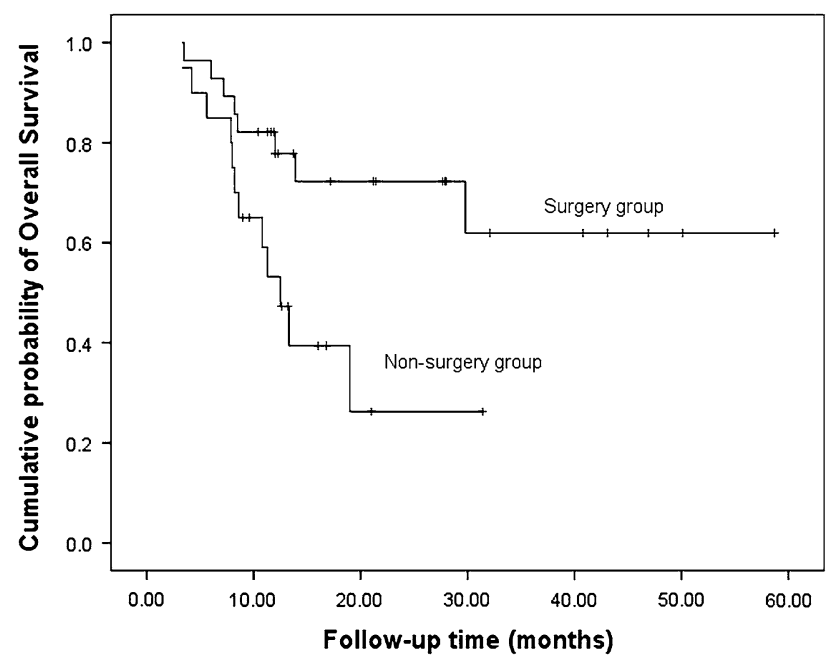

Fig. 4 Overall survival of surgery group and non-surgery group

Table 4 Toxicities of chemotherapy $(N=48)$

\begin{tabular}{lrrrr}
\hline Toxicities & 1 & 2 & 3 & 4 \\
\hline Leukocytopenia & 14 & 7 & 5 & 0 \\
Thrombocytopenia & 9 & 2 & 2 & 0 \\
Anemia & 3 & 1 & 1 & 0 \\
Nausea/vomiting & 8 & 5 & 1 & 0 \\
Diarrhea & 5 & 3 & 0 & 0 \\
Hand-foot skin reaction & 12 & 3 & 0 & 0 \\
Hepatic dysfunction & 5 & 0 & 0 & 0 \\
Neuropathy & 10 & 3 & 0 & 0 \\
\hline
\end{tabular}

five patients had grade 3 leukopenia. No patients had grade 4 toxicities. One patient had an acute perforation of stomach 5 days after the first cycle of XELOX regimen. Only one of the 28 patients had postoperative complications described as lung infection after surgery.

\section{Discussion}

Patients with gastric cancers and PAN are currently considered to have distant metastatic disease and cannot be cured by surgery. However, the prognosis and clinical manifestations are somewhat different compared with other sites of metastasis. Gastric cancer patients with PAN involvement alone were found to have better survival than other advanced gastric cancer patients with multiple organ sites metastasis [6]. Therefore, it did not seem logical to simply regard gastric cancer with PAN as M1 disease and receive only palliative treatments. We have shown that the survival data in patients after palliative chemotherapy who did not receive surgery was 12.5 months which is similar to that found in other studies [6]. However, in the current study, patients who had prior chemotherapy, and subsequent radical gastrectomy, had more prolonged survival than the patients who could not have operation.

Whether surgery could be done, when surgery should be done and what kind of lymph node dissection should be done are the most demanding questions in the current study. In the current study design, we defined patients with PAN metastasis as those who had PAN $>1 \mathrm{~cm}$ in diameter by abdominal CT scanning as an inclusion criterion. False positive findings may be caused by inflammatory lymphadenopathy. CT criteria for assessing nodal metastases are based on nodal size and shape, and the presence of central necrosis. Nodal size criteria can be used when nodes are homogeneous and clearly delineated. The generally used definition of a metastatic lymph node depends mostly on a greatest nodal diameter of more than $1 \mathrm{~cm}$. With this definition, the correlation with the pathological diagnosis has been reported to be close to $80 \%$ [13]. Therefore, when PAN metastasis disappeared or shrank to $<1.0 \mathrm{~cm}$, radical surgery was considered. Gastrectomy with D2 lymphadenectomy is the standard treatment for curable gastric cancer in eastern Asia, and outcomes of patients with D2 lymphadenectomy plus PAND were not significantly better than those after D2 lymphadenectomy alone in the patients with metastasis at PAN [5]. Sasako et al. reported that compared with D2 lymphadenectomy alone, treatment with D2 lymphadenectomy plus para-aortic nodal dissection did not improve survival rates in curable gastric cancer. Gastrectomy with D2 lymphadenectomy is the standard treatment for curable gastric cancer in eastern Asia. Therefore, we adopted the D2 lymphadenectomy for all patients without para-aortic lymphadectomy. In the current study, in the seven patients who relapsed after radical operation, two 
had local recurrences, and five had distant metastases to lung, bone and left supraclavicular lymph node. None of them had PANs involvement, indicating that the gastric cancer patients with PAN metastasis could be cured by D2 lymphadenectomy and neoadjuvant chemotherapy. As the patients who received radical surgery had PAN metastasis disappear or shrink to $<1.0 \mathrm{~cm}$ as determined by radiological evaluation before operation, adjuvant radiotherapy was not needed after D2 lymphadenectomy,

D2 resection is safe and effective, but the safety of D2 resection after chemotherapy had not been evaluated. In the current study, all 24 patients who received R0 resection had a D2 lymphadenectomy. The median time from surgery to discharge was 9 days, which is similar to that in patients who were not treated with neoadjuvant chemotherapy [14]. Despite one trial published by Schuhmacher et al. [15] suggesting a significantly higher risk of mortality after neoadjuvant chemotherapy, most of the randomized studies did not confirm this, as highlighted in a recent meta-analysis [16]. In the current study, only one of the 28 patients had postoperative complication which was described as a lung infection after surgery. D2 lymphadenectomy after preoperative chemotherapy is safe and effective.

An existing study showed that patients who benefitted most from neoadjuvant chemotherapy were those who achieved a complete response (pCR) with no residual microscopic tumor. However, achievement of pCR has been reported to be uncommon, occurring in only $10-15 \%$ of patients [17], while clinical responses have been reported to range from 32 to $42 \%$ [17-20]. In the current study, the CR rate was $10.7 \%$, and the clinical response rate was $49 \%$, which is similar to the results of other studies [1720]. Three patients had pCR had partial clinical responses determined radiologically which indicates that $\mathrm{CT}$ imaging results do not always agree with histological findings.

Potentially resectable gastric cancer has been treated by several regimens of combination chemotherapy. Due to the results of the MAGIC trial [7] and Real-2 trial [21], epirubicin, cisplatin and 5-Fu (ECF) and epirubicin, cisplatin and capecitabine (EOX) are considered to be standard perioperative chemotherapy in Western countries. Among the various combination chemotherapy regimens that are currently being investigated to treat advanced gastric cancer, oxaliplatin and capecitabine (XELOX) appears to be useful and have encouraging antitumor activity [22]. Phase 2 studies have demonstrated that XELOX produces a favorable tumor response rate with a relatively mild toxicity profile [23]. The current study showed that the regimen of XELOX is safe and without severe adverse effects. The limitations of this study include small sample size and single research center data.

In conclusion, gastric cancer patients with PAN involvement can benefit from pre-surgical chemotherapy and subsequent radical surgery with D2 lymphadenectomy.
Although few advanced gastric cancer patients have PAN involvement alone, the current results provide a practical treatment plan for this special group of patients. Large scale, multicenter, and randomized trials will help to further determine the best treatment strategy for these patients.

Acknowledgments The authors appreciate very much the great effort of the staff members in the Department of Medical Oncology at Fudan University Zhongshan Hospital for their helpful suggestions and assistance.

Conflict of interest The authors of the manuscript declare that there are no conflicts of interest.

Open Access This article is distributed under the terms of the Creative Commons Attribution License which permits any use, distribution, and reproduction in any medium, provided the original author(s) and the source are credited.

\section{References}

1. Forman D, Burley VJ (2006) Gastric cancer: global pattern of the disease and an overview of environmental risk factors. Best Pract Res Clin Gastroenterol 20(4):633-639

2. Baba M, Hokita S, Natsugoe S et al (2000) Paraaortic lymphadenectomy in patients with advanced carcinoma of the upper third of the stomach. Hepatogastroenterology 47(33):893-896

3. Japanese Gastric Cancer Association (2011) Japanese classification of gastric carcinoma: 3rd English edition. Gastric Cancer 14(2):101-112

4. Edge SB, Compton CC (2010) The American Joint Committee on Cancer: the 7th edition of the AJCC cancer staging manual and the future of TNM. Ann Surg Oncol 17(6):1471

5. Sasako M, Sano T, Yamamoto S et al (2008) D2 lymphadenectomy alone or with para-aortic nodal dissection for gastric cancer. N Engl J Med 359(5):453-462

6. Park IH, Kim SY, Kim YW et al (2011) Clinical characteristics and treatment outcomes of gastric cancer patients with isolated para-aortic lymph node involvement. Cancer Chemother Pharmacol 67(1):127-136

7. Cunningham D, Allum WH, Stenning SP et al (2006) MAGIC trial participants. Perioperative chemotherapy versus surgery alone for resectable gastroesophageal cancer. N Engl J Med 355(1):11-20

8. Nakajima T, Ota K, Ishihara S et al (1997) Combined intensive chemotherapy and radical surgery for incurable gastric cancer. Ann Surg Oncol 4(3):203-208

9. Yonemura Y, Sawa T, Kinoshita K et al (1993) Neoadjuvant chemotherapy for high-grade advanced gastric cancer. World J Surg 17(2):256-262

10. Eisenhauer EA, Therasse P, Bogaerts J et al (2009) New response evaluation criteria in solid tumours: revised RECIST guideline (version 1.1). Eur J Cancer 45(2):228-247

11. Becker K, Mueller JD, Schulmacher C et al (2003) Histomorphology and grading of regression in gastric carcinoma treated with neoadjuvant chemotherapy. Cancer 98(7):1521-1530

12. Trotti A, Colevas AD, Setser A et al (2003) CTCAE v3.0: development of a comprehensive grading system for the adverse effects of cancer treatment. Semin Radiat Oncol 13(3):176-181

13. Som PM (1992) Detection of metastasis in cervical lymph nodes: CT and MR criteria and differential diagnosis. AJR Am J Roentgenol 158(5):771-773 
14. Marcus SG, Cohen D, Lin K et al (2003) Complication of gastrectomy following CPT-11-based neoadjuvant chemotherapy for gastric cancer. J Gastrointest Surg 7(8):1015-1023

15. Schuhmacher C, Gretschel S, Lordick F et al (2010) Neoadjuvant chemotherapy compared with surgery alone for locally advanced cancer of the stomach and cardia: European Organisation for Research and Treatment of Cancer randomized trial 40954. J Clin Oncol 28(35):5210-5218

16. Liao Y, Yang ZL, Peng JS, Xiang J, Wang JP (2013) Neoadjuvant chemotherapy for gastric cancer: a meta-analysis of randomized, controlled trials. J Gastroenterol Hepatol 28(5):777-782

17. Lowy AM, Mansfield PF, Leach SD et al (1999) Response to neoadjuvant chemotherapy best predicts survival after curative resection of gastric cancer. Ann Surg 229(3):303-308

18. Hartgrink HH, van de Velde CJ, Putter H et al (2004) Neo-adjuvant chemotherapy for operable gastric cancer: long term results of the Dutch randomized FAMTX trial Include. Eur J Surg Oncol 30(6):643-649
19. Nio Y, Koike M, Omori H et al (2004) A randomized consent design trial of neoadjuvant chemotherapy with tegafur plus uracil (UFT) for gastric cancer-a single institute study. Anticancer Res 24(3b): 1879-1887

20. Luo HY, Xu RH, Wang F et al (2010) Phase II trial of XELOX as first-line treatment for patients with advanced gastric cancer. Chemotherapy 56(2):94-100

21. Cunningham D, Okines AF, Ashley S (2008) Capecitabine and oxaliplatin for advanced esophagogastric cancer. N Engl J Med 358(1):36-46

22. Van Meerten E, Eskens FA, Van Gameren EC, Van Der Gaast A (2007) First-line treatment with oxaliplatin and capecitabine in patients with advanced or metastatic oesophageal cancer: a phase II study. Br J Cancer 96(9):1348-1352

23. Liu C, Sun Q, Hang X, Zhong B, Wang D (2008) Multicenter phase II study of capecitabine plus oxaliplatin as a first-line therapy in Chinese patients with advanced gastric cancer. Anticancer Drugs 19(8):825-831 